\title{
An HCG-rich microenvironment contributes to ovarian cancer cell differentiation into endothelioid cells in a three-dimensional culture system
}

\author{
MIN SU $^{1 *}$, CHAO FAN $^{1,3^{*}}$, SAINAN GAO $^{1}$, AIGUO SHEN $^{2}$, XIAOYING WANG $^{2}$ and YUQUAN ZHANG ${ }^{1}$ \\ ${ }^{1}$ Department of Obstetrics and Gynecology, The Affiliated Hospital of Nantong University; \\ ${ }^{2}$ The Immunology Laboratory of Nantong University, Nantong, Jiangsu 226001, P.R. China
}

Received April 11, 2015; Accepted July 2, 2015

DOI: $10.3892 /$ or.2015.4215

\begin{abstract}
We investigated the expression of human chorionic gonadotropin (HCG) and its effects on vasculogenic mimicry (VM) formation in ovarian cancer cells under normoxic and hypoxic conditions in three-dimensional matrices preconditioned by an endothelial-trophoblast cell co-culture system. The co-culture model was established using human umbilical vein endothelial cells (HUVECs) and HTR-8 trophoblast cells in a three-dimensional culture system. The co-cultured cells were removed with $\mathrm{NH}_{4} \mathrm{OH}$, and ovarian cancer cells were implanted into the preconditioned matrix. VM was identified morphologically and by detecting vascular markers expressed by cancer cells. The specificity of the effects of exogenous HCG in the microenvironment was assessed by inhibition with a neutralizing anti-HCG antibody. HCG siRNA was used to knock down endogenous HCG expression in OVCAR-3 ovarian cancer cells. HTR-8 cells 'fingerprinted' HUVECs to form capillary-like tube structures in co-cultures. In the preconditioned HCG-rich microenvironment, the number of vessel-like network structures formed by HCG receptorpositive OVCAR-3 cells and the expression levels of CD31, VEGF and factor VIII were significantly increased. The preconditioned HCG-rich microenvironment significantly increased the expression of hypoxia inducible factor- $1 \alpha$ $(\mathrm{HIF}-1 \alpha)$ and VM formation in OVCAR-3 cells under hypoxic
\end{abstract}

Correspondence to: Professor Yuquan Zhang or Dr Sainan Gao, Department of Obstetrics and Gynecology, The Affiliated Hospital of Nantong University, 20 Xisi Road, Nantong, Jiangsu 226001, P.R. China

E-mail: zhangyuquan2011@126.com

E-mail: sumin_nt@126.com

Present address: ${ }^{3}$ The Affiliated Changshu Hospital of Suzhou University, 1 Shuyuan Street, Changshu, Jiangsu 215500, P.R. China

*Contributed equally

Key words: co-culture system, preconditioned microenvironment, human chorionic gonadotropin, ovarian cancer, vasculogenic mimicry, hypoxia conditions. Treatment with a neutralizing anti-HCG antibody but not HCG siRNA significantly inhibited the formation of vessel-like network structures. HCG in the microenvironment contributes to OVCAR-3 differentiation into endothelioid cells in three-dimensional matrices preconditioned with an endothelial-trophoblast cell co-culture system. HCG may synergistically enhance hypoxia-induced vascular markers and HIF-1 $\alpha$ expression. These findings would provide perspectives on new therapeutic targets for ovarian cancer.

\section{Introduction}

Vasculogenic mimicry (VM) is the conversion of aggressive cancer cells to an endothelial cell-like phenotype and subsequent formation of tumor cell-lined vasculature. Thus, tumors can form their own vasculature for nourishment through VM, independent of host blood vessels. The presence of VM correlates with an increased risk of metastasis and therefore poor clinical outcome (1). VM has been reported in many malignant tumor types, including melanoma, liver, prostate and breast cancer, glioma and renal cancer (2-7); yet what fascinates us most is the discovery of VM in the most lethal gynecologic malignancy, ovarian cancer (8).

The etiology of VM remains unclear. A dynamic, complex relationship exists between VM and the microenvironment, which plays a pivotal role in cancer progression and has been shown to affect VM formation in melanoma (9). Hypoxia, a feature of the tumor microenvironment, promotes VM formation in three-dimensional cultures (10-12). Morphological analysis of choriocarcinoma demonstrated that central blood channels were surrounded by neoplastic trophoblastic cells rather than endothelial cells. In the periphery of choriocarcinoma, tumor cells invaded uterine stroma-derived blood vessels, where trophoblastic cells replaced endothelial cells, forming anastomoses between endothelium-lined vessels and trophoblast-lined pseudovascular channels. These findings indicated that choriocarcinoma, noted for its high level of human chorionic gonadotropin (HCG) secretion, is among the few tumor types that utilize VM (13). However, little is known about the role of HCG in VM.

HCG is a cytokine that is ectopically expressed in a variety of malignant tumor microenvironments, including ovarian 
cancer, endometrial carcinoma, cervical, seminomatous testicular, bladder and breast cancer (14-20). HCG expression in ovarian cancer tissue varies relative to grade and stage. HCG acts on the luteinizing hormone (LH)/HCG receptor (LH-R), and HCG expression correlates with LH-R expression in ovarian cancer tissue which has prognostic value (21). Ziecik et al (22) demonstrated ubiquitous HCG protein expression in ovarian cancer samples, and at least $40 \%$ of epithelial ovarian cancer expressed LH-R at high levels. Co-expression of the HCG protein and LH-R in cancer cells may indicate an autocrine or paracrine mechanism of tumor-derived HCG activity in the tumor microenvironment. HCG has now been proposed as a novel angiogenic factor that could mediate angiogenic pathways $(23,24)$.

Our recent study showed that treatment of ovarian cancer cell line OVCAR-3 with recombinant HCG resulted in formation of tumor cell-lined vasculature and significantly increased vascular marker expression (25). Herein, we extend our previous study to determine the influence of the trophoblast cell microenvironment on VM in ovarian cancer. To further explore a possible effect of HCG secreted by trophoblast cells on VM in ovarian cancer cells, we developed an endothelial-trophoblast cell co-culture system as a preconditioned, HCG-enriched microenvironment. After the co-cultured cells were removed (26), HCG receptor-positive OVCAR-3 cells or HCG receptor-negative SKOV3 ovarian cancer cells were implanted into the preconditioned matrix. VM was identified morphologically and by detection of vascular cell markers expressed by cancer cells.

Hypoxia mediates tumor VM through hypoxia inducible factor-1 $\alpha(\mathrm{HIF}-1 \alpha)(27)$, and ovarian cancer cells form vasculogenic-like networks in a three-dimensional culture under hypoxic conditions (10). We further used the dual cell system under hypoxic conditions to gain insight into the interaction between HCG and hypoxia in promoting VM in ovarian cancer.

\section{Materials and methods}

Cell culture. Human umbilical vein endothelial cells (HUVECs) were purchased from Cambrex (East Rutherford, NJ, USA) and maintained in Dulbecco's modified Eagle's medium (DMEM) growth media supplemented with $8 \%$ fetal bovine serum. The first trimester human extravillous trophoblast cell line HTR-8 and the ovarian cancer OVCAR-3 and SKOV3 cell lines were purchased from the American Type Culture Collection (ATCC; Manassas, VA, USA) and cultured in 1640 growth medium. OVCAR-3 cells were incubated under normoxic $\left(21 \% \mathrm{O}_{2}\right)$ or hypoxic $\left(1 \% \mathrm{O}_{2}\right)$ conditions. Culture dishes in the hypoxia group were placed in a manually controlled airtight chamber and hypoxic conditions were created by flushing $5 \% \mathrm{CO}_{2}$ and $94 \%$ nitrogen through the GENbox jar 2.5L chamber (bioMérieux SA, Japan) until the $\mathrm{O}_{2}$ concentration was reduced to $1 \%$, as measured by a mini oxygen meter.

Establishment of an endothelial-trophoblast cell co-culture system and preconditioned microenvironment. Undiluted Matrigel was plated into 24-well tissue culture plates at $300 \mu \mathrm{l} /$ well and polymerized for $30 \mathrm{~min}$ at $37^{\circ} \mathrm{C}$. HUVECs $\left(1.0 \times 10^{5}\right)$ and HTR-8 $\left(1.0 \times 10^{5}\right)$ cells were stained with the green fluorescent linker dye PKH67 and the red fluorescent linker dye PKH26, respectively, and then co-cultured in the Matrigel. The formation of tube-like structures was monitored by fluorescence microscopy. After establishment of the endothelial-trophoblast cell co-culture system for three days, co-cultured cells were removed with $20 \mathrm{mM} \mathrm{NH}_{4} \mathrm{OH}$ to establish the preconditioned microenvironment. Furthermore, ovarian cancer OVCAR-3 and SKOV3 cells were planted in the preconditioned Matrigel.

ELISA. After establishing the co-culture system, the expression of HCG in the supernatant was investigated at $0,24,48$, 72 and 96 h by ELISA using an HCG ELISA kit (Beijing North Institute of Biological Technology, Beijing, China). Assay diluent RD1N (50 $\mu \mathrm{l})$ and $50 \mu \mathrm{l}$ of each sample or standard were mixed in the wells of a microplate coated with a polyclonal antibody specific for $\beta-\mathrm{HCG}$, and then $100 \mu \mathrm{l}$ of substrate solution was added. The enzyme reaction was terminated with the addition of a diluted hydrochloric acid solution. The intensity of the color was measured at a wavelength of $450 \mathrm{~nm}$. The specificity of the effects of exogenous HCG in the microenvironment was further assessed by complete inhibition with a $2.4 \mu \mathrm{g} / \mathrm{ml}$ neutralizing anti-HCG antibody.

Light and electron microscopy. Ovarian cancer cells were examined by an inverted phase contrast microscope (Carl Zeiss, Inc., Thornwood, NY, USA). For scanning electron microscopy, cells were fixed in $2.5 \%$ cold glutaraldehyde in $0.1 \mathrm{M}$ sodium cacodylate buffer and post-fixed in $1 \%$ osmium tetroxide. Specimens were dehydrated in a graded series of ethanol, displaced, critically point-dried and sputtercoated with gold before scanning electron microscopy analysis (S-520; Hitachi, Tokyo, Japan).

Real-time polymerase chain reaction (PCR). Reverse transcription (RT) of RNA was performed using the TaqMan Gold RT-PCR kit as described by the manufacturer (Applied Biosystems, Foster City, CA, USA). Real-time quantitative PCR was performed with the Applied Biosystems 9700HT sequence detection system. Each target was amplified in triplicate with an $\mathrm{HCG}$-specific primer probe set. We devised primers as follows: HIF-1 $\alpha, 5$ '-CTGCCACCACTGATGA ATTA-3' and 5'-GTATGTGGGTAGGAGATGGA-3'; LH-R, 5'-TGGCTGCTGTAAACGTCGGG-3' and 5'-GGAGAGC TGTACCTTGACAGTGC-3'; CD31, 5'-ATTGCAGTGGTTA TCATCGGAGTG-3' and 5'-CTCGTTGTTGGAGTTCAGA AGTGG-3'; factor VIII (VWF), 5'-ATCGAGGGTCTCG GGGATGCG-3' and 5'-TGCGAAGAGTGCTGCGAAT GCT-3'; VEGFA, 5'-GACATCTTCCAGGAGTACC-3' and 5'-TGCTGTAGGAAGCTCATCTC-3'; $\beta$-HCG, 5'-TGCCAC CCTGGCTGTGGA-3' and 5'-GCGGTAGTTGCACACCAC CTG-3'. A 5-fold titration of control template was included along with RT-negative and no-template controls. Relative mRNA levels were calculated using the standard curve method (ABI User Bulletin no. 2) with $18 \mathrm{~S}$ as the endogenous control and $0 \mathrm{~h}$ as the calibrator.

Western blot analysis. The mouse monoclonal antibodies raised against VEGF, VWF, HIF- $1 \alpha, \beta-H C G$ and LH-R were obtained from Santa Cruz Biotechnology (Santa Cruz, CA, 

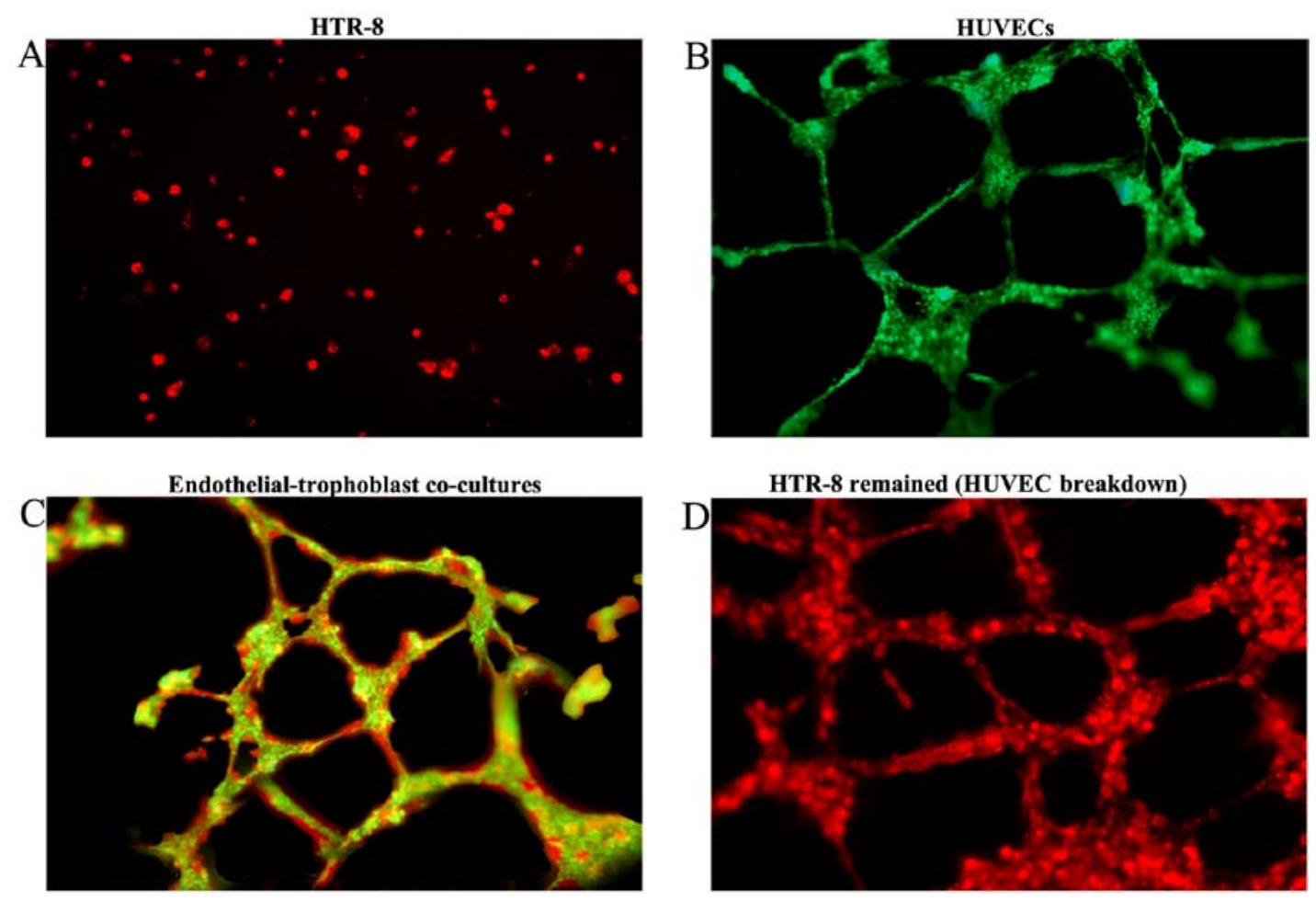

Figure 1. Representative fluorescent images of endothelial-trophoblast co-cultures on Matrigel. Human umbilical vein endothelial cells (HUVECs) and trophoblast HTR-8 cells labeled with green fluorescent PKH67 and red fluorescent PKH26 cell trackers, respectively, were independently cultured (A and B) or co-cultured (C and D) on Matrigel. Capillary-like tube structures were observed for HUVECs (B) yet not for HTR-8 cells (A). However, in the co-culture system, HTR-8 cells 'fingerprinted' the HUVECs to form capillary-like tube structures (C). After co-culturing for $72 \mathrm{~h}$, HUVECs in the co-culture system began to break down, and only the tube structures formed by HTR-8 cells remained (D). (A-D) Original magnification, x10.

USA). The rabbit monoclonal antibody raised against CD31 was purchased from Bioworld (Dublin, OH, USA). Cellular proteins were isolated, separated on an $8 \%$ SDS-tris polyacrylamide gel and were transferred to a PVDF membrane. The membranes were blocked before incubation with primary antibodies (1:100) and horseradish peroxidase-conjugated secondary antibody $(1: 1,000)$. Immunocomplexes were visualized by electrochemiluminescence. Protein expression was semi-quantified using a Tiannen imager and analysis system (Shanghai, China).

Small interfering RNA (siRNA). HCG- $\beta$ siRNAs were synthesized and ligated into a pcDNA ${ }^{\mathrm{TM}}$ 6.2-GW/EmGFPmiR vector (Invitrogen, Carlsbad, CA, USA). The sequences of pSilencer/HCG- $\beta$ were: AGCAGCAACAGCAGCAGCCTC. Transfections were performed with $3 \mu \mathrm{g}$ silencing plasmid pcDNA $^{\mathrm{TM}} 6.2-\mathrm{GW} /$ EmGFP-CGB5 miR, $9 \mu \mathrm{g}$ ViraPower Packaging Mix and $2 \mu \mathrm{l}$ Lipofectamine 2000 (Invitrogen). Control cells were mock-transfected. Stably transfected cells were selected by $0.4 \mathrm{mg} / \mathrm{ml} \mathrm{G} 418$ (Merck, Darmstadt, Germany) after two weeks.

Indirect immunofluorescence. Cultured cells were fixed in $4 \%$ paraformaldehyde then incubated with $2 \%$ BSA. After blocking, the sections were incubated with mouse anti-human LH-R/HCG antibody (Santa Cruz Biotechnology). Sections were stained with 1:1,000 FITC-conjugated goat anti-mouse IgG1 secondary antibody (Caltag). Nuclei were visualized by staining with 4',6-diamidino-2-phenylindole (DAPI). Sections were imaged by immunofluorescence microscopy. Negative controls were produced by omitting the primary antibody.

Statistical analysis. The results are presented as the mean \pm SD (standard deviation). Data were analyzed using SPSS 16.0 for Windows (SPSS, Inc., Chicago, IL, USA). One-way ANOVA analysis was performed to identify differences.

\section{Results}

Establishment of an endothelial-trophoblast cell co-culture system and preconditioned microenvironment. We established an endothelial-trophoblast cell co-culture system on Matrigel. First trimester trophoblast HTR-8 cells failed to form tube-like structures even after $72 \mathrm{~h}$ of incubation and remained aggregated as lumps or acinar structures (Fig. 1A). Under identical culture conditions, HUVECs migrated, polarized, underwent cytoskeleton remodeling, branched and formed the classical capillary-like tube (Fig. 1B). However, when the two cell types were co-cultured, HTR-8 cells spontaneously 'fingerprinted' the capillary tube-like structures of the HUVECs (Fig. 1C). After $72 \mathrm{~h}$ of co-culturing, HUVECs began to breakdown, and only the tube structures formed by HTR- 8 cells remained. HTR-8 cells distributed along the walls of the tubes and achieved complete replacement of the HUVECs (Fig. 1D). After establishing the endothelial-trophoblast cell co-culture system for three days, co-cultured cells implanted in the matrix were removed with $\mathrm{NH}_{4} \mathrm{OH}$ to establish the preconditioned microenvironment. 
A
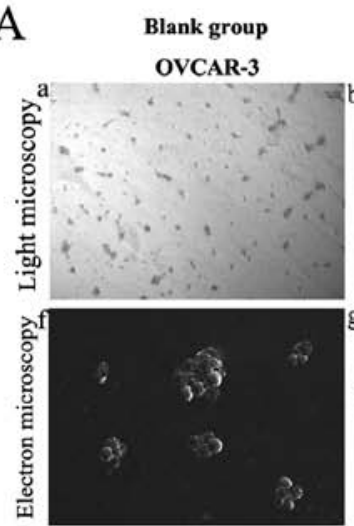

B

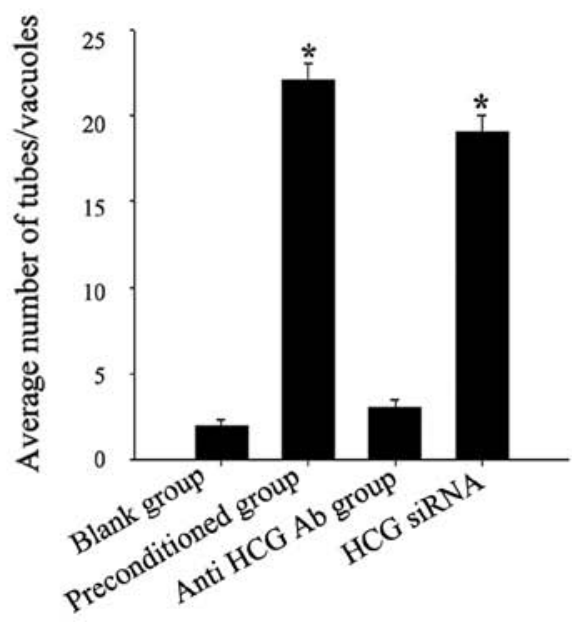

Anti HCG Ab group

OVCAR-3

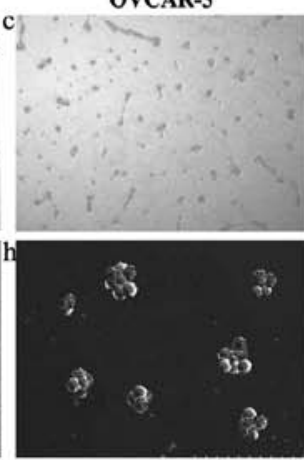

$\mathrm{C}$
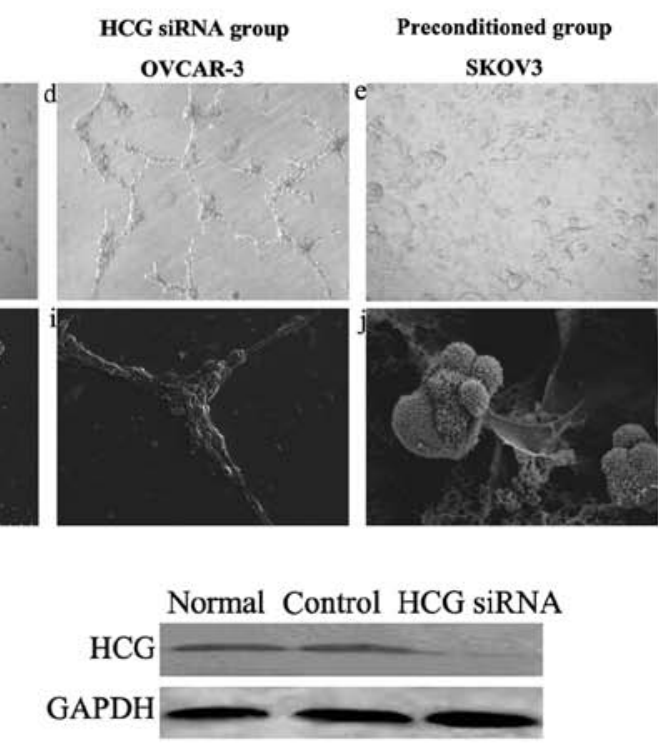

$\mathrm{D}$

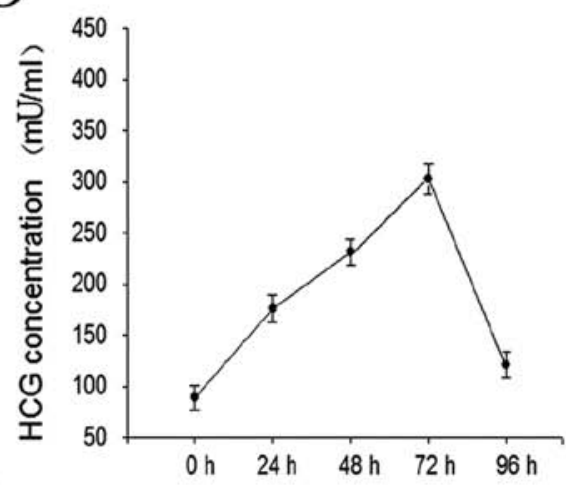

Figure 2. Role of the human chorionic gonadotropin (HCG)-rich microenvironment in regulating vasculogenic mimicry (VM) formation. (A) Morphological flexibility of ovarian cancer cells. (a-e) Light microscopy and (f-j) scanning electron microscopy images. The morphological plasticity of OVCAR-3 ovarian cancer cells was absent on a normal three-dimensional Matrigel (a and f). The presence of tubular networks and formation of channels was observed in OVCAR-3 cells in preconditioned Matrigel (b and g) and was inhibited by treatment with a neutralizing anti-human chorionic gonadotropin (HCG) antibody (c and h). Downregulation of HCG expression using HCG siRNA on OVCAR-3 cells failed to decrease vasculogenic mimicry (VM) formation (d and i). SKOV3 ovarian cancer cells failed to form the capillary-like tubes in preconditioned Matrigel (e and j). a-e, Original magnification, $x 50$; f-j, $\mathrm{x} 400$. (B) The average number of tubes/vacuoles formed by OVCAR-3 cells was quantified in four different fields (magnification, $\mathrm{x} 4$ ). The number of vessel-like network structures formed by OVCAR-3 cells was significantly increased in preconditioned Matrigel. * $\mathrm{p}<0.05$. (C) HCG siRNA vector was transfected with OVCAR-3. The protein expression in OVCAR-3 cells was efficiently knocked down. Normal, blank control; control, the control vector transfected cells; HCG RNAi, HCG knockdown cells. (D) The levels of HCG were measured by ELISA at the indicated times.

Morphological flexibility of OVCAR-3 cells induced by the HCG-rich microenvironment. OVCAR-3 cells aggregated as lumps on normal Matrigel (Fig. 2A-a and -f) and migrated, polarized, underwent cytoskeleton remodeling, branched and formed classical capillary-like tubes on the preconditioned Matrigel (Fig. 2A-b and -g). The number of vessel-like network structures formed by OVCAR-3 cells was significantly increased in the preconditioned microenvironment (Fig. 2B). However, SKOV3 cells failed to form capillary-like tubes (Fig. 2A-e and $-\mathrm{j}$ ).

We detected expression of HCG in the liquid supernatant collected after co-culturing for $0,24,48,72$ and 96 h. HCG expression increased gradually, peaked at $72 \mathrm{~h}$, then began to decrease but remained higher than normal levels (Fig. 2D). Tube formation was significantly inhibited in the OVCAR-3 cells treated with the neutralizing anti-HCG antibody added to the endothelial-trophoblast cell co-culture in Matrigel $(\mathrm{p}<0.05)$. This result indicated that HCG in the microen- vironment was critical for VM formation in OVCAR-3 cells (Fig. 2A-c and -h).

OVCAR-3 ovarian cancer cells were previously reported to secrete HCG in an autocrine manner (15). To clarify the source of HCG, we used HCG siRNA to knockdown endogenous expression of HCG in OVCAR-3 cells (Fig. 2C). We found that HCG siRNA treatment in OVCAR-3 cells failed to block VM formation in the preconditioned microenvironment (Fig. 2A-d and -i). This result indicated that exogenous HCG secreted by trophoblasts may promote VM formation in OVCAR-3 cells.

HCG-induced expression of LH-R and vascular cell markers in OVCAR-3 cells. OVCAR-3 ovarian cancer cell line expresses LH-R (28), which is consistent with our immunofluorescence results (Fig. 3A). HCG exerts its effects by binding to LH-R. Western blotting and PCR results indicated upregulation of LH-R expression in OVCAR-3 cells in the co-culture 


\section{A}

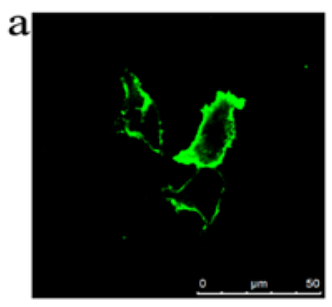

LH-R

B

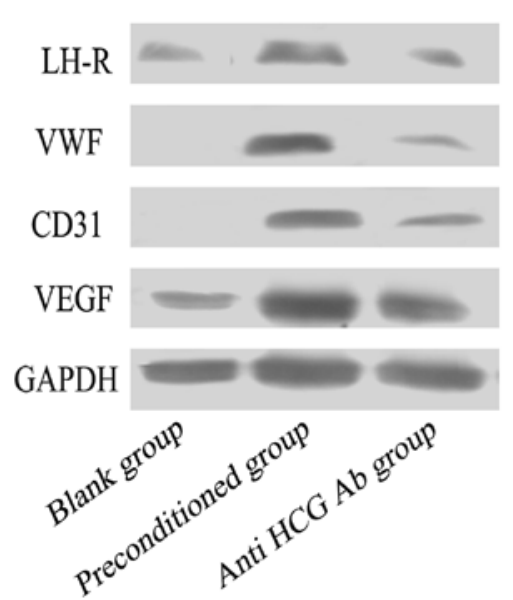

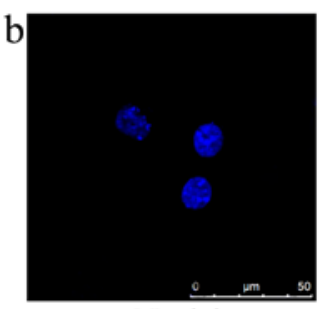

Nuclei

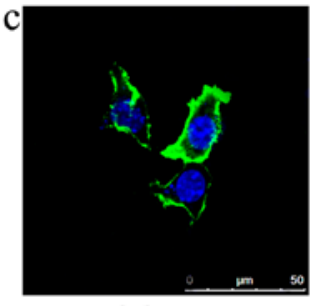

Merge
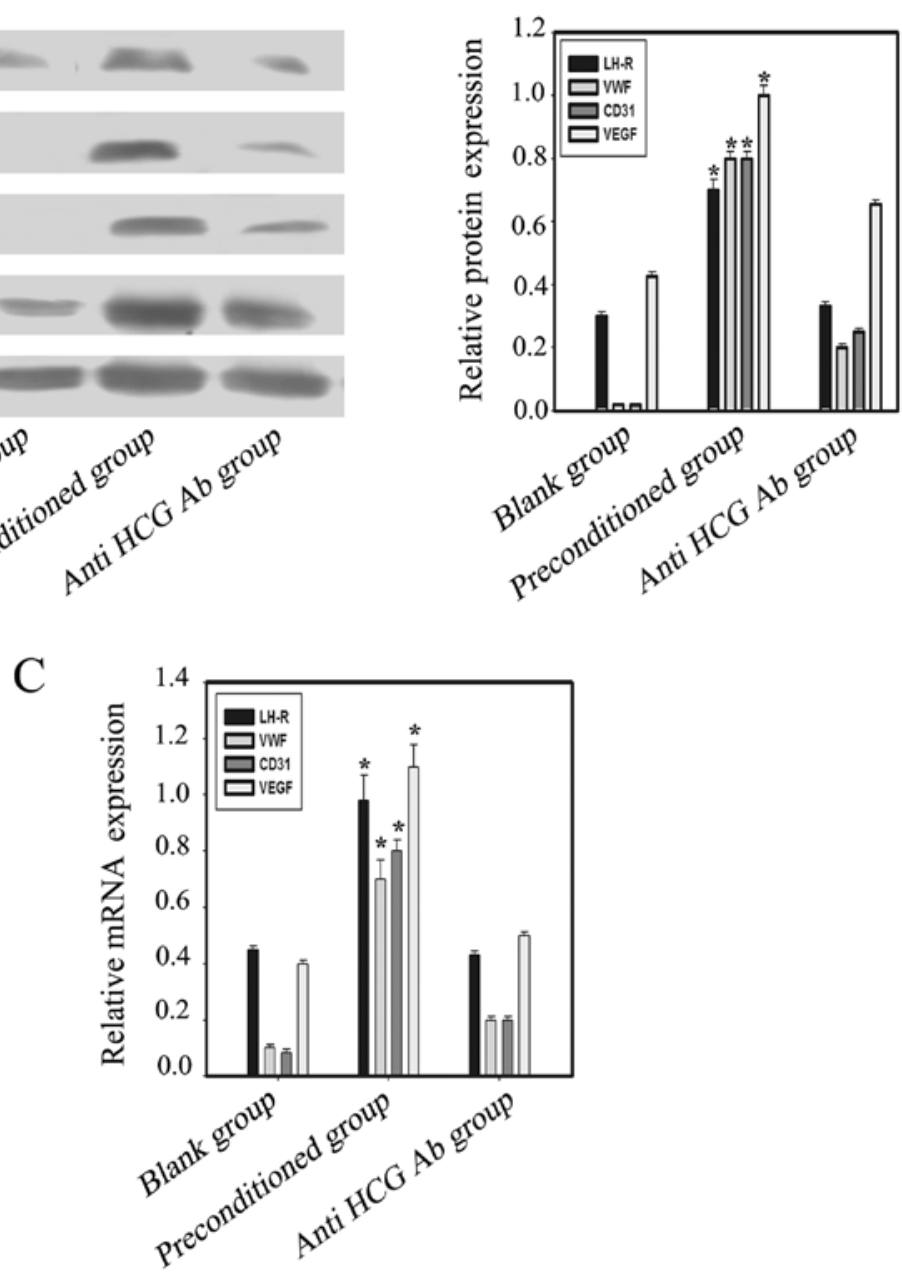

Figure 3. Expression of the luteinizing hormone (LH)/human chorionic gonadotropin (HCG) receptor (LH-R) and vascular cell markers in OVCAR-3 ovarian cancer cells induced by the HCG-rich microenvironment. (A) Immunofluorescence images of OVCAR-3 cells. Blue, DAPI-stained nuclei; green, LH-R. (B and C) Western blotting and real-time PCR indicated significant increases in the protein and mRNA levels of LH-R and vascular markers in the preconditioned group compared with the blank group, and the effect of HCG in the environment was inhibited by the neutralizing anti-HCG antibody. ${ }^{*} \mathrm{p}<0.05$.

environment. Furthermore, the expression of the vascular markers CD31, VEGF and VWF in the preconditioned group was significantly increased when compared with the blank and anti-HCG antibody groups ( $\mathrm{p}<0.05)$ (Fig. 3B and C).

The role of the HCG-rich microenvironment in regulating VM formation and HIF-1 $\alpha$ expression under hypoxic conditions. Under hypoxic conditions, OVCAR-3 cells were implanted in normal or preconditioned Matrigel. Our data showed that the morphological flexibility, i.e., the appearance of network structures and channels, of OVCAR-3 cells on the preconditioned Matrigel was significantly greater than that of untreated cells on normal Matrigel. This plasticity was largely inhibited by a neutralizing anti-HCG antibody. The expression of the vascular markers in OVCAR-3 cells implanted in precondi- tioned Matrigel was also significantly higher than in the blank and anti-HCG antibody groups (Fig. 4A and C). The peak level of HCG was significantly higher under hypoxic conditions than normoxia (Fig. 4B).

Furthermore, we detected the expression of HIF-1 $\alpha$ in the three groups. Our data showed a significantly increased amount of HIF-1 $\alpha$ expression in the preconditioned group compared with the other two groups, indicating possible synergistic effects of HCG and hypoxia (Fig. 4D and E).

\section{Discussion}

Vasculogenic mimicry (VM) is found in many highly invasive tumors and may greatly influence tumor metastasis and recurrence (29). VM provides a new perspective on antitumor 
A
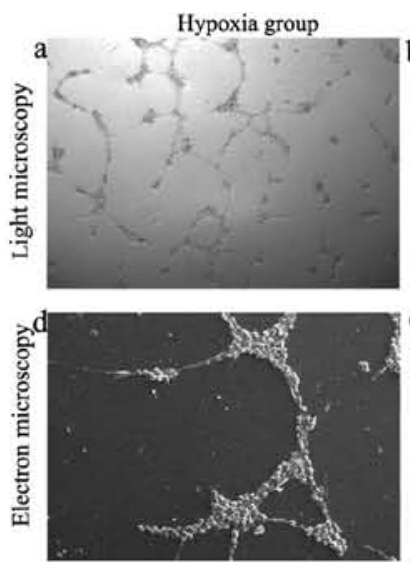

B

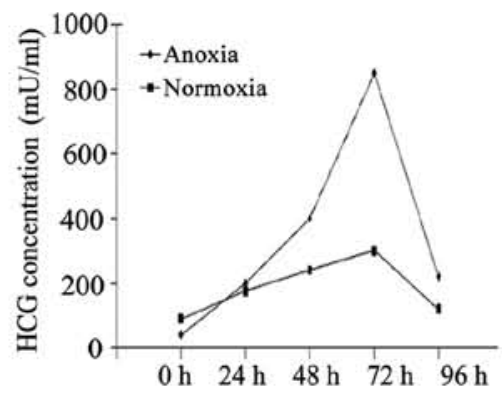

Coculture time of HUVEC and HTR-8 under hypoxia condition

$\mathrm{D}$

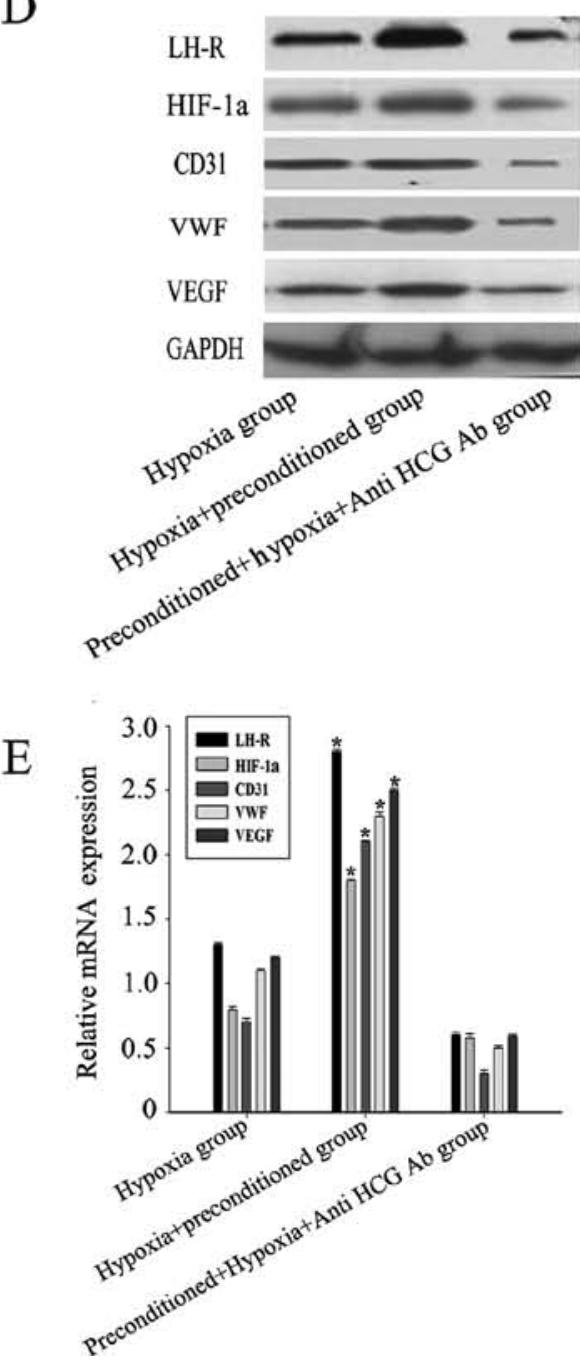

Hypoxia+preconditioned group

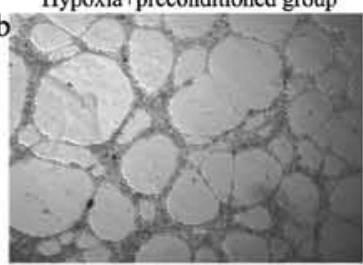

Hypoxia+preconditioned +anti HCG Ab
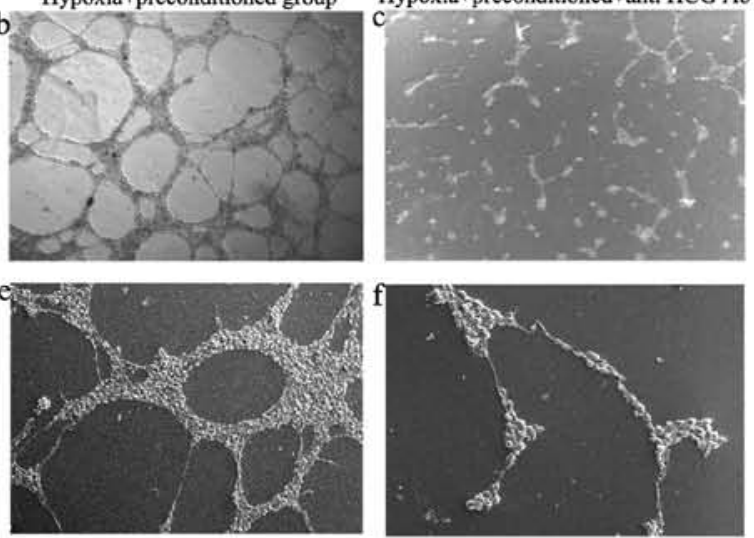

$\mathrm{C}$
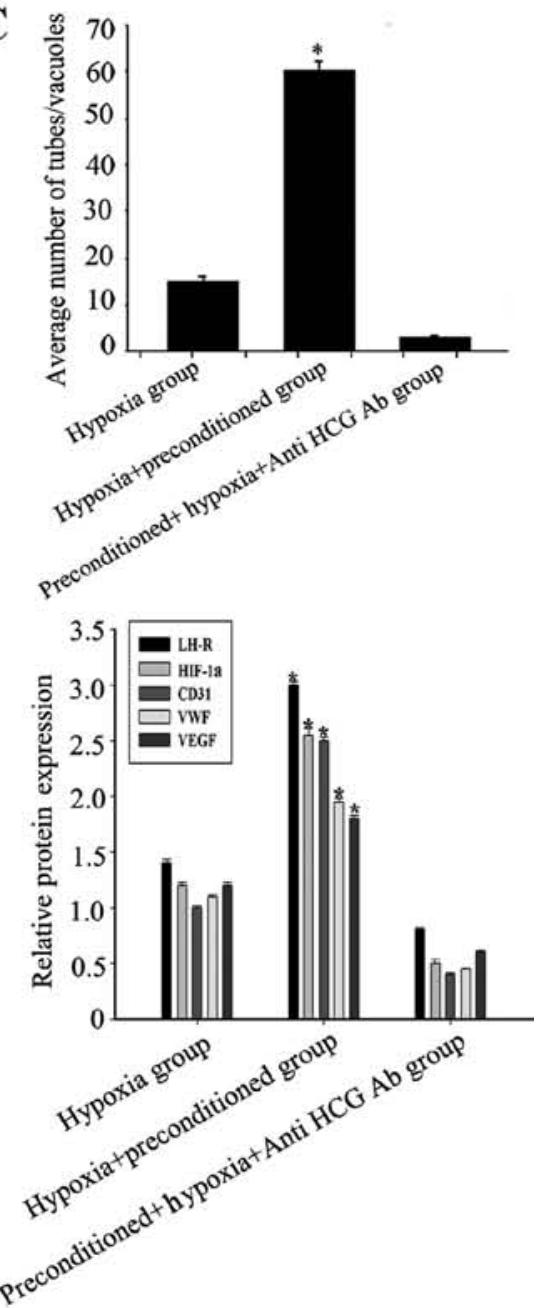

Figure 4. The role of the human chorionic gonadotropin (HCG)-rich microenvironment in regulating vasculogenic mimicry (VM) formation under hypoxic conditions. (A) Morphological flexibility of OVCAR-3 ovarian cancer cells. (a-c) Light microscopy and (d-f) scanning electron microscopy images. OVCAR-3 cells displayed atypical tubular networks and channels in a three-dimensional gel exposed hypoxic $\left(1 \% \mathrm{O}_{2}\right)$ conditions (a and d). The formation of tubular networks and channels was significantly increased when OVCAR-3 cells were implanted on preconditioned Matrigel under hypoxic conditions ( $\mathrm{b}$ and e) and were significantly inhibited by treatment with a neutralizing anti-HCG antibody under hypoxic conditions ( $c$ and f). a-c, Original magnification, x50; d-f, x200. (B) The levels of HCG were measured by ELISA at the indicated times. The peak level of HCG was significantly higher under hypoxic conditions than normoxic conditions. (C) The average number of tubes/vacuoles formed by OVCAR-3 cells was quantified in four different fields (magnification, $x 4$ ). (D and E) The protein and mRNA levels of the vascular markers and HIF-1 $\alpha$ in the preconditioned group increased significantly compared with the other two groups. " $\mathrm{p}<0.05$. 
therapy and has become a hot topic and difficult problem in gynecological cancer research. The interaction between tumors and other cell types, i.e., the tumor microenvironment, is thought to play an important role in VM. However, the molecular details governing the microenvironment remain poorly understood. Seftor et al (26) investigated the unique epigenetic effects of the microenvironment of aggressive melanoma cells on the behavior of non-aggressive melanoma cells. Collagen matrices preconditioned with aggressive melanoma cells capable of forming VM-induced poorly aggressive melanoma cells, which are initially unable to form VM, to express vasculogenic genes and form VM in vitro. In this process, the factors secreted by tumor cells or other niche components in the microenvironment play a critical role.

$\mathrm{HCG}$, a recently identified angiogenic factor, is secreted ectopically in the microenvironment of some non-trophoblastic tumors. It not only stimulates growth and inhibits apoptosis of cancer cells (30), yet also has a role in angiogenesis by increasing capillary formation and endothelial cell migration in vivo and in vitro $(31,32)$. HCG has been regarded as a stage- and grade-independent prognostic factor that identifies a subgroup of patients with increased risk of aggressive disease (33). Elevated serum or tissue levels of $\beta$-HCG are frequently associated with higher cancer aggressiveness and resistance to therapy (34), while the reduction of $\beta$-HCG expression by modified U1 snRNA caused apoptosis in cervical cancer cells (19).

Notably, the sinus in choriocarcinoma is similar to VM formation in ovarian cancer. Trophoblast cells, which produce high levels of HCG, invade the endovascular tube and replace the endothelial cells lining the vessels, a process known as vascular remodeling. In the present study, we designed a three-dimensional endothelial-trophoblast cell co-culture system as a preconditioned $\beta$-HCG-rich microenvironment mimicking vascular remodeling to further investigate the role of $\beta$-HCG on ovarian cancer cell differentiation into endothelioid cells.

We found that OVCAR-3 cells formed VM in the microenvironment preconditioned by the endothelial-trophoblast cell co-culture system. The fundamental events in the process were not clearly defined, and any contribution of HCG to VM formation in the microenvironment was unknown. To clarify the role of HCG from the microenvironment in VM formation, we measured the concentration of HCG in the preconditioned microenvironment. After $72 \mathrm{~h}$ of co-culture, we measured up to $4,000 \mathrm{mU} / \mathrm{ml} \beta$-HCG in the microenvironment, and the network structures formed by OVCAR-3 were inhibited by a neutralizing anti-HCG antibody. Moreover, in preconditioned Matrigel, CD31, VEGF and VWF expression levels were upregulated in the OVCAR-3 cells, and these inductive effects were mostly neutralized by treatment with an anti-HCG antibody, which was verified by western blotting and real-time PCR. The relative protein expressions of vascular markers were still slightly higher in anti-HCG antibody group than in blank group from our data, possibly since that the neutralizing anti-hCG antibody failed to recognize all the dimeric forms of the hormone. However, LH-R-negative SKOV3 ovarian cancer cells could not form VM under identical conditions. These results indicated that $\mathrm{HCG}$ secreted in the microenvironment contributes to OVCAR-3 differentiation into endothelioid cells.
Hypoxia induces HIF-1 $\alpha$ expression and formation of VM (35). Under hypoxic conditions, HIF-1 $\alpha$ is the main regulator of cancer cell transcriptional responses to hypoxia and VM channels $(35,36)$. We verified this finding herein by analyzing HIF-1 $\alpha$ expression and further investigated the possible relationship between hypoxia and an HCG-rich preconditioned microenvironment. When the preconditioned Matrigel was placed under hypoxic conditions, the number and length of VM were significantly increased compared with the normoxic condition. Moreover, the expression levels of HCG, LH-R and HIF-1 $\alpha$, and the vascular markers were also significantly increased compared with the hypoxia group. These results indicated that HCG and hypoxia may have synergistic effects on ovarian cancer cell differentiation into endothelioid cells.

Nearly $90 \%$ of advanced-stage ovarian cancer patients develop recurrence and eventually die from the development of resistance to cytotoxic chemotherapy. Therefore, there is an urgent need to develop novel therapeutics that selectively kill ovarian cancer cells (37). Since there is abundant LH-R expression in numerous epithelial ovarian cancers, LH-R may become an attractive therapeutic target. Vaccines against $\beta$-HCG have been successfully demonstrated, suggesting a potential adjuvant therapy in cancer treatment (38). Our results demonstrate the powerful influence of the HCG-rich endothelial-trophoblast microenvironment on endothelial differentiation and VM formation in OVCAR-3 ovarian cancer cells. We anticipate that these findings may provide new perspectives on tumor cell plasticity and offer new targets for novel therapeutic strategies. In addition, further investigation is required to clarify the signal transduction pathways involved in HCG-induced OVCAR-3 ovarian cancer cell differentiation.

\section{Acknowledgements}

We thank the Laboratory of Immunology, Nantong University, China for technical assistance. The present study was supported by grants from the National Natural Science Foundation of China (30801226). The manuscript was edited by the professional language editing service Elsevier Webshop.

\section{References}

1. Vartanian AA, Stepanova EV, Gutorov SL, Solomko ESH, Grigorieva IN, Sokolova IN, Baryshnikov AY and Lichinitser MR: Prognostic significance of periodic acid-Schiff-positive patterns in clear cell renal cell carcinoma. Can J Urol 16: 4726-4732, 2009.

2. Maniotis AJ, Folberg R, Hess A, Seftor EA, Gardner LM, Pe'er J, Trent JM, Meltzer PS and Hendrix MJ: Vascular channel formation by human melanoma cells in vivo and in vitro: Vasculogenic mimicry. Am J Pathol 155: 739-752, 1999.

3. Sun D, Sun B, Liu T, Zhao X, Che N, Gu Q, Dong X, Yao Z, Li R, Li J, et al: Slug promoted vasculogenic mimicry in hepatocellular carcinoma. J Cell Mol Med 17: 1038-1047, 2013.

4. Qin L, Ren Y, Chen AM, Guo FJ, Xu F, Gong C, Cheng P, Du Y and Liao H: Peroxisome proliferator-activated receptor $\gamma$ ligands inhibit VEGF-mediated vasculogenic mimicry of prostate cancer through the AKT signaling pathway. Mol Med Rep 10: 276-282, 2014.

5. El Hallani S, Boisselier B, Peglion F, Rousseau A, Colin C, Idbaih A, Marie Y, Mokhtari K, Thomas JL, Eichmann A, et al: A new alternative mechanism in glioblastoma vascularization: Tubular vasculogenic mimicry. Brain 133: 973-982, 2010. 
6. Francescone RA III, Faibish M and Shao R: A Matrigel-based tube formation assay to assess the vasculogenic activity of tumor cells. J Vis Exp 7: 3040, 2011.

7. Zhang Y, Sun B, Zhao X, Liu Z, Wang X, Yao X, Dong X and Chi J: Clinical significances and prognostic value of cancer stem-like cells markers and vasculogenic mimicry in renal cell carcinoma. J Surg Oncol 108: 414-419, 2013.

8. Sood AK, Fletcher MS, Zahn CM, Gruman LM, Coffin JE, Seftor EA and Hendrix MJ: The clinical significance of tumor cell-lined vasculature in ovarian carcinoma: Implications for anti-vasculogenic therapy. Cancer Biol Ther 1: 661-664, 2002.

9. Chen LX, Sun BC, Zhang SW, He YJ, Li XR and He ZJ: The mechanisms of microenvironments influence on vasculogenic mimicry between intraocular and subcutaneous melanoma. Zhonghua Yan Ke Za Zhi 45: 641-646, 2009 (In Chinese).

10. Yao LQ, Feng YJ, Ding JX, Jing HM, Xu CJ, Chen SF, Su M and Yin LH: Characteristics and differentiated mechanism of vascular endothelial cells-like derived from epithelial ovarian cancer cells induced by hypoxia. Int J Oncol 30: 1069-1075, 2007.

11. Du J, Sun B, Zhao X, Gu Q, Dong X, Mo J, Sun T, Wang J, Sun R and Liu Y: Hypoxia promotes vasculogenic mimicry formation by inducing epithelial-mesenchymal transition in ovarian carcinoma. Gynecol Oncol 133: 575-583, 2014.

12. Su M, Feng YJ, Yao LQ, Cheng MJ, Xu CJ, Huang Y, Zhao YQ and Jiang $\mathrm{H}$ : Plasticity of ovarian cancer cell SKOV3ip and vasculogenic mimicry in vivo. Int J Gynecol Cancer 18: 476-486, 2008.

13. Shih IeM: Trophoblastic vasculogenic mimicry in gestational choriocarcinoma. Mod Pathol 24: 646-652, 2011.

14. Muller CY and Cole LA: The quagmire of hCG and hCG testing in gynecologic oncology. Gynecol Oncol 112: 663-672, 2009.

15. Szajnik M, Nowak-Markwitz E, Szczepański MJ and Spaczyński M: Assessment of expression of luteinizing hormone (LH)/human chorionic gonadotropin (hCG) receptor (LH/hCGR) and hCG protein in ovarian cancer tissues. Ginekol Pol 78: 939-943, 2007 (In Polish).

16. Lempiäinen A, Stenman UH, Blomqvist $\mathrm{C}$ and Hotakainen $\mathrm{K}$ : Free beta-subunit of human chorionic gonadotropin in serum is a diagnostically sensitive marker of seminomatous testicular cancer. Clin Chem 54: 1840-1843, 2008.

17. Iles RK, Delves PJ and Butler SA: Does hCG or hCG $\beta$ play a role in cancer cell biology? Mol Cell Endocrinol 329: 62-70, 2010.

18. Burczynska B, Booth MJ, Iles RK, Shah A, Shiled A and Butler SA: Stable knockdown of hCG $\beta$ mRNA expression in bladder cancer cells results in significant growth inhibition. Anticancer Res 33: 3611-3614, 2013.

19. Jankowska A, Gunderson SI, Andrusiewicz M, Burczynska B Szczerba A, Jarmolowski A, Nowak-Markwitz E and Warchol JB: Reduction of human chorionic gonadotropin beta subunit expression by modified U1 snRNA caused apoptosis in cervical cancer cells. Mol Cancer 7: 26, 2008.

20. Jankowska AG, Andrusiewicz M, Fischer N and Warchol PJ: Expression of hCG and GnRHs and their receptors in endometrial carcinoma and hyperplasia. Int J Gynecol Cancer 20: 92-101, 2010

21. Lenhard M, Tsvilina A, Schumacher L, Kupka M, Ditsch N, Mayr D, Friese K and Jeschke U: Human chorionic gonadotropin and its relation to grade, stage and patient survival in ovarian cancer. BMC Cancer 12: 2, 2012.

22. Ziecik AJ, Kaczmarek MM, Blitek A, Kowalczyk AE, Li X and Rahman NA: Novel biological and possible applicable roles of LH/hCG receptor. Mol Cell Endocrinol 269: 51-60, 2007.
23. Pietrowski D, Wiehle P, Sator M, Just A and Keck C: Regulation of the angiopoietin-2 gene by hCG in ovarian cancer cell line OVCAR-3. Horm Metab Res 42: 328-333, 2010.

24. Brouillet S, Hoffmann P, Chauvet S, Salomon A, Chamboredon S, Sergent F, Benharouga M, Feige JJ and Alfaidy N: Revisiting the role of hCG: New regulation of the angiogenic factor EG-VEGF and its receptors. Cell Mol Life Sci 69: 1537-1550, 2012.

25. Su M, Wei W, Xu X, Wang X, Chen C, Su L and Zhang Y: Role of hCG in vasculogenic mimicry in OVCAR-3 ovarian cancer cell line. Int J Gynecol Cancer 21: 1366-1374, 2011.

26. Seftor EA, Meltzer PS, Kirschmann DA, Margaryan NV, Seftor RE and Hendrix MJ: The epigenetic reprogramming of poorly aggressive melanoma cells by a metastatic microenvironment. J Cell Mol Med 10: 174-196, 2006.

27. Zhao N, Sun BC, Sun T, Ma YM, Zhao XL, Liu ZY, Dong XY, Che N, Mo J and Gu Q: Hypoxia-induced vasculogenic mimicry formation via VE-cadherin regulation by Bcl-2. Med Oncol 29: 3599-3607, 2012

28. Gebauer G, Mueller N,Fehm T, Berkholz A,Beck EP, Jaeger W and Licht P: Expression and regulation of luteinizing hormone/human chorionic gonadotropin receptors in ovarian cancer and its correlation to human chorionic gonadotropin-doxorubicin sensitivity. Am J Obstet Gynecol 190: 1621-1628, 2004.

29. Sun Q, Zou X, Zhang T, Shen J, Yin Y and Xiang J: The role of miR-200a in vasculogenic mimicry and its clinical significance in ovarian cancer. Gynecol Oncol 132: 730-738, 2014.

30. Elzarrad K, Haroon A, Reed D and Al-Mehdi AB: Early incorporated endothelial cells as origin of metastatic tumor vasculogenesis. Clin Exp Metastasis 26: 589-598, 2009.

31. Michel RM, Aguilar JL and Arrieta O: Human chorionic gonadotropin as an angiogenic factor in breast cancer during pregnancy. Med Hypotheses 68: 1035-1040, 2007.

32. Phan B, Rakenius A, Pietrowski D, Bettendorf H, Keck C and Herr D: hCG-dependent regulation of angiogenic factors in human granulosa lutein cells. Mol Reprod Dev 73: 878-884, 2006.

33. Lempiäinen A, Hotakainen $\mathrm{K}$, Blomqvist $\mathrm{C}$, Alfthan $\mathrm{H}$ and Stenman UH: Hyperglycosylated human chorionic gonadotropin in serum of testicular cancer patients. Clin Chem 58: 1123-1129, 2012.

34. Crabb SJ, Sharp A, Head J, Chau C, Wheater M, Geldart TR, et al: Investigating serum $\beta$-HCG as an independent prognostic factor in patients (pts) receiving chemotherapy $(\mathrm{Ct})$ for transitional cell carcinoma (TCC) of the urothelial tract. J Clin Oncol 31: 4549, 2013.

35. Sun W, Shen ZY, Zhang H, Fan YZ, Zhang WZ, Zhang JT, $\mathrm{Lu} X \mathrm{XS}$ and Ye C: Overexpression of HIF-1 $\alpha$ in primary gallbladder carcinoma and its relation to vasculogenic mimicry and unfavourable prognosis. Oncol Rep 27: 1990-2002, 2012.

36. Comito G, Calvani M, Giannoni E, Bianchini F, Calorini L, Torre E, Migliore C, Giordano S and Chiarugi P: HIF-1 $\alpha$ stabilization by mitochondrial ROS promotes Met-dependent invasive growth and vasculogenic mimicry in melanoma cells. Free Radic Biol Med 51: 893-904, 2011.

37. McCann GA, Rath KS, Naidu S, Lata P, Hemant B, Sudhakar M, Hideg K, Houghton P, Kuppusamy P, Cohn DE, et al: HO-3867, is selectively cytotoxic to ovarian cancer cells through a dual mechanism of action involving the STAT3 and AKT pathways. Cancer Res 73 (Suppl 8): 1039, 2013.

38. Yang J, Zhang Y, Wang H, Gao Z, Wang Z, Liu B, Zhang X, Du M, Huang X, Xu M, et al: Vaccination with the repeat $\beta$-hCG C-terminal peptide carried by heat shock protein-65 (HSP65) for inducing antitumor effects. Tumour Biol 33: 1777-1784, 2012. 